\title{
Biological Concept of Bacterial Pathogenicity (Theoretical Review)
}

\author{
Yurii V. Ezepchuk \\ 2937 South Revere St., Aurora, CO, USA \\ Email:ezepchuk@usa.net
}

How to cite this paper: Ezepchuk, Y.V. (2017) Biological Concept of Bacterial Pathogenicity (Theoretical Review). Advances in Microbiology, 7, 535-544.

https://doi.org/10.4236/aim.2017.77042

Received: May 3, 2017

Accepted: June 27, 2017

Published: June 30, 2017

Copyright $\odot 2017$ by authors and Scientific Research Publishing Inc. This work is licensed under the Creative Commons Attribution International License (CC BY 4.0).

http://creativecommons.org/licenses/by/4.0/

\begin{abstract}
Biological nature of the bacterial pathogenicity phenomenon is based on the interaction of prokaryotic and eukaryotic organisms. The phenomenon is the poly-functional biological potency of germs that are realized by factors (determinants) of pathogenicity. Some fundamental biological functions are responsible for bacterial pathogenicity in a multi-cellular host organism: the adhesive function, the function of invasion and penetration into the cell, the function of evasion of host defense, and the damage function. The action of adhesion, invasion and evasionis directed to towards establishing an ecological niche in multi-cellular host while the aim of the damaging function is destruction of the environment.
\end{abstract}

\section{Keywords}

Bacterial Pathogenicity, Biological Function, "Pathogenicity Islands", Infectious Diseases, Prokaryotic and Eukaryotic Organisms

\section{Introduction}

For a long time, the prevailing approach to the study of pathogenicity phenomenon was focusing on identification of specific antigenic determinants in bacterial pathogens. It enabled scholars to identify and characterize antigen features of a microbial cell but it could not provide any information about its biological function which is critical to understanding the phenomenon of pathogenicity. The situation was changed drastically when modern techniques of molecular biology became available and were used to determine individual pathogenic factors, their functional properties, and corresponding molecular structures [1]. Systematic analysis of experimental and theoretical data obtained for the last decades, furthered accumulation the similar functionally active bio-molecules in separate groups which became the foundation of the modern understanding of bacterial pathogenicity [2]. 
Definitions: Phenomenon of pathogenicity is the polyfunctional biological potency of germs that are realized by factors (determinants) of pathogenicity.

Bacterial pathogenicity is an ability of bacteria to induce and develop infectious diseases in multi-cellular organisms (human, animals and plants).

Virulence is a degree of pathogenicity measured by the in vivo (LD50) and in vitro (ID50) tests (highly virulent, weakly virulent and non-virulent strains).

Pathogenic factors (determinants) are the bio-molecules produced by pathogen and are responsible for interaction with the host tissue cells.

"Pathogenicity Islands" are the bacterial genome mobile elements that carry genes encoding the pathogenicity factors production.

\section{Adhesive Function}

It is known that the first step of interaction bacterial pathogen and cells of multicellular host organism starts from adhesive process. Many pathogens possess the special morphological structures that are located on the cell surface and are called fimbria or common pili [3]. These organelles are responsible for adhesion of pathogen to the host tissue cells. Despite their diversity in structure and biogenesis, pili/fimbriaes typically consist of a long fiber formed by homopolymerised subunits or pilins, and accessory pilins that often function as adhesions [4]. Some bacteria can produce nonpilus adhesive structures that mediate specific adherence to host tissues. The majority of nonpilus adhesions are proteins, but other structures such as lipopolysaccharides (LPS) and lipoteichoic acids also have adhesive function [5], Table 1.

Among the adhesive macromolecules, some show high affinity to the process of physic chemical binding with eukaryotic cells. The ligand-receptor interaction of bacterial adhesions and the host cell surface demonstrates a high degree of specificity. For example, certain pathogenic bacteria infect only certain species of animals, e.g. Enteropathogenic E. coli K-88 infections are limited to pigs; E. coli K-99 strain infects calves. Other pathogens such as Neisseria $s p$ and $E$. coli CFA I and CFA II infect humans; Group A streptococcal infections occur only in humans [6] [7].

Biological features of some spore pathogens are belonging to Bacillus and Clostridium family showed that these bacteria have reduced adhesive function or not at all. Vegetable cells generated by spores that infected host organism immediately start production of extracellular toxin responsible for damaging function [8].

Table 1. Components responsible for adhesive function.

\begin{tabular}{ccc}
\hline Pathogen & Morphological structure & Somatic components \\
\hline Gram-negative bacteria spp & Fimbriae/pili & Lipopolysacharide (LPS) \\
Staphylococcus $\mathrm{spp}$ & Lipoteichoic acids (LTA) \\
Streptococcus spp & Protein M (?) \\
Lactobacillus $\mathrm{spp}$ & Protein (?) \\
Micrococcus $\mathrm{spp}$ & Protein (?) \\
\hline
\end{tabular}


After adherence to a cell surface, microorganisms begin to reproduce intensively and increase their population while colonizing parts of the host tissue. Besides the very important role in colonization of the host tissue, some adhesions are also involved in cell aggregation, biofilm formation, DNA uptake, phage transduction and gliding motility [4].

From point of view of evolutionary process, the appearance of microorganisms that could interact with living cells of multi-cellular tissues can be considered as a first step in the establishment of a new ecological space. Microbial cells that possessed such adhesive properties in relation to animal or plant tissues became commensals and potential pathogens. Natural selection completed formation of the new species and contributed to the promotion of the specificity of adhesive function. The adhesive function lead to extend microbial population in multi-cellular environment and it means that it was a new property allowing new germ species to broaden their habitat [9].

\section{Function of Invasion and Cell Penetration}

Another function that was responsible to spread bacterial pathogens in multicellular media was the ability to penetrate intercellular space or invasive capacity. Some extracellular bacteria can employ the enzymes that their saprophyte predecessors used to degrade organic remains in water and soil. For example, enzymes such as hyaluronidase, lecithinase, proteases and some glycopeptidases which produced by Clostridium pathogens were able to split intercellular concreted compounds and invade the tissue [2] [10].

In process of evolution and during interaction with eukaryotic cells, some bacterial pathogens acquired the capacity to penetrate inside host cells. Most invading bacteria enter the host cell by using either a triggered or a zippered mechanism. Special bacteria-derived effectors are able to induce membrane ruffles into the eukaryotic cell and to activate a clathrin mediated structure through which bacteria enter the cell. At the entry site, activated signaling pathways regulated the fate of the invading microorganism. Bacteria may then replicate in either cytoplasmic or vacuolar niches [10]. Vacuoles are acting membranebounded compartments have unique specificity to a given bacteria in its composition and behavior. Under the action of intracellular pathogens their vacuoles transform into a favorable niche where they undermine the anti-bacterial host defense mechanisms. This is mainly achieved through the action of bacterial proteins (effectors) that are translocated out of the vacuoles into the host cytoplasm [11] [12] [13].

The invasive and penetrative function is another property of the pathogenicity complex, in addition to selective adhesion to host eukaryotic cells that led to occupy new ecological space.

\section{Function of Evasion of Host Defense}

To develop infectious process, the entered pathogen needs to defeat the action of the nonspecific immune system machinery. This essential component is the 
compliment system. Compliment activation (through the classical, the lectin, and the alternative pathways) tags microbes for destruction by phagocytic cells causing microbial lysis. Many pathogens have developed strategies to evade the complement system [7] [14].

Some pathogenic bacteria possess special morphological structures (capsules) or biochemical features which allow them to resist the main lines of host internal defense against them. The capsule is located on the surface of a bacterial cell and has a gelatinous consistence usually reinforced by chains or threads of linear polymers. To resist compliment activation and absorption by phagocytes, the capsule of microbial cells needed certain chemical traits. For example, the vegetative forms of Bacillus anthracis that had a capsule consisting of $D$-isomers glutamic acid became invulnerable to host phagocytes because it is known that the $D$-amino acids isomers peptides are resistant to the action of proteolytic degradation [9].

Many pathogens, however, produce diverse biochemical features which are responsible for evasion the complement system. These bacterial substances had different molecular and chemical structure. This group of pathogenicity factors mainly consists of peptides or proteins. Thus, staphylococcal protein A and streptococcal protein M, LPS produced by Gram-negative bacteria, glycoproteins and other mixed polymers are included in the group. Some pathogens such as Streptococcus pyogenes, Staphylococcus aureus and Treponema pallidum use fibronectin binding proteins to provide an antigenic disguise if they clotted fibrin on the cell surface to avoid host defenses. Pathogenic Mycobacterium has a waxy cell wall that resists attack or digestion by most tissue bactericides. Intact LPS of some Gram-negative pathogens may protect the bacterial cells from complement-mediated lysis or the action of lysozyme [6] [14].

Despite different mechanisms of resistance to the host cell activity, these substances played the same functional role: they protected microbial germs from host non-immune and immune system (Table 2).

Recently, an original function of S-layer proteins as a defense against antibacterial peptides has been demonstrated. The defense peptides of host are small

Table 2. Cell components responsible for evasion function.

\begin{tabular}{cc}
\hline Pathogen & Component \\
\hline Pneumococcus $\mathrm{sp}$ & capsule polysaccharide \\
Bacillus anthracis & capsule D-glutamile polypeptide \\
Pseudomonas aeruginosa & slime polysaccharide \\
Staphylococcus aureus & Protein A \\
Streptococcus $\mathrm{sp}$ & Protein $\mathrm{M}$ \\
Trepanema pallidum & Fibronectin binding protein (FBP) \\
Staphylococcus aureus & FBP \\
Streptococcus pyogenes & FBP \\
Mycobacterium sp & waxy cell \\
Yersinia pestis & capsule protein F-1 \\
\hline
\end{tabular}


cationic, amphypathic molecules produced all organisms as a first line of defense against microbial invasion and are found at host-microbe interfaces, such epithelial layers. Initially it was established that the role of the peptides was to control microbial levels through direct antimicrobial activity. Actually, mechanisms of bacterial resistance to S-layer proteins are not yet clear [14] [15].

One of the pathogenicity factors with evasion function is the ability that also can alter host cell apoptosis or cause other forms of cell death [16].

Thus, all three biological functions played a very important role in the colonization of the new ecological niche by microbial pathogen and guaranteed its necessary life in the host organism. At this stage, pathogen was devoid of aggressive features and did not induce any specific damage to human or animal multicellular systems. This type of interaction between the prokaryotic pathogen and the eukaryotic tissue cells of the host organism can be characterized as a kind of a symbiotic like balance [9].

\section{Damaging Function}

The pathogenicity complex was further complicated after the damaging function developed in the pro-pathogens, which possessed the above mentioned properties. The tox-genes appeared in the bacterial genome were responsible for production of aggressive substances directed to induce various types of dysfunction in the host tissue cells. According to chemical structure and mechanism of damaging action, bacterial toxins can be divided to two categories: endo- and exo-toxins. Depends on specific biological activity of toxic molecules, the host target cells can be undergoing by direct or indirect mechanism of destructive action [17] (Table 3).

Endotoxons (LPS) of Gram-negative bacteria are constituents of the outer membrane of the bacterial cell wall. Toxicity of the cell unbound LPS is associated with the lipid component activity (Lipid A). Lipid $A$ is known to react at the surfaces of macrophages causing them to release tumor necrosis factor (TNF- $\alpha$ ) and probably other cytokines. In contrast to the protein exotoxins, endotoxins do not act enzymatically and they are less potent and less specific in their action. We can assume that the induction of lipid A cytokines play the leading role in the indirect mechanism of damaging action developed by endotoxins. Blood and lymphoid cells as well as immune system cells and compliment system, are targets that undergo endotoxin action [16] [18].

Table 3. Components responsible for damaging function.

\begin{tabular}{cc}
\hline Direct action toxin type & Indirect actiontoxin type \\
\hline 1. Poreforning toxins: & Lipid A (Gram-negative LPS) \\
leukocidins, hemolysins & Superantigenic toxins: \\
2. AB subunit toxins: & Staphylococcal enterotoxins, TSST \\
A subunit-affect cyclic AMP & Streptococcal pyrogenic toxins \\
A subunit-inactivate protein synthesis & \\
B subunit-receptor binding function & \\
\hline
\end{tabular}


Besides endotoxins, some Gram-negative pathogens can produce exo-toxin proteins. For example, enteropathogenic E. coli strains synthesized extra-cellular LT- and ST-enterotoxins or cytolytic molecules [10].

A family of staphylococcal and streptococcal exotoxins, such as enterotoxins, toxic shock syndrome toxin (TSST), pyrogenic toxins and others, belong to the group of toxic bio-molecules with indirect mechanism of damaging action and they are named superantigens. These toxins are represented by peptide molecules that have the distinct domain structure. They are able to elicit massive activation of the $T$ lymphocytes but induce weakly antibody response in the host organism. The molecular mechanism of the $T$ cell proliferation is realized by interaction with ClassII MHC molecules on APCs and specific VB chains of the $T$ cell receptor. The process of activation under the action of superantigens results to production of lymphokines such as $I L-1, T N F$ and others [19].

Thus, we can suppose that endotoxins of Gram-negative bacteria and superantigen exotoxins produced by Gram-positive pathogens have similar mechanism of indirect damaging action on the host immune system.

Another group of damaging macro-molecules is represented by toxin exoproteins that were able to inactivate or to destroy the vitally important physiological systems in the organ tissue cells by direct way. Both Gram-positive and Gram-negative bacteria produce soluble protein toxins. Most exotoxins possess an enzymatic activity that can be realized in contact with the host tissue target cells. Bacterial protein exotoxins are different in their molecular structure; some of them are represented by simple polypeptide molecules and others have a complicated subunit structure [20].

Certain simple protein toxins have broad cytotoxic activity and cause both very specific as well as nonspecific damage of tissue cells. Pore-forming mechanism of action underlies cytotoxicity of some exotoxins, such as hemolysins and leukocidins produced by both Gram-positive and Gram-negative bacteria. The essential functional feature of cytolysins is their capacity to provoke the formation of hydrophobic pores in the cytoplasmic membranes of target eukaryotic cells. The process results from the binding of the toxins with membrane receptor on the cell surface, followed by their oligomerization, which lead to insertion of the oligomers into the membrane and formation of protein-lined channels. This insertion provokes the impairment of the osmotic balance of the cell and subsequent cytolysis [21].

The peculiarity of so-called "chimera" toxin proteins is that they have both hormone-like and enzyme-like properties. Molecular model of the "chimera" toxin complex is sometimes described as a $A+B$ (?) formula where the subunit $B$ is the peptide (or peptides) responsible for the membrane receptor binding, and the subunit " $A$ " is the peptide that is able to penetrate the cell and damage the intracellular target. The hormone-like component of the macromolecule was able to recognize specific membrane receptors on the sensitive tissue cell and bind to the cell surface. After the ligand-receptor binding, the enzymatic active components of the bifunctional structure were subjected to endocytosis or 
pore-forming mechanism and targeted one of the vital systems of the host cell [20]. For instance, the active enzyme subunit A of toxins produced by Vibrio cholerae, Bordetella pertussis and Bacillus anthracis can affect cyclic AMP and disturb a critical regulatory process of the host tissue cell. These above mentioned pathogens are completely nonrelated bacteria but their toxins function in a similar way, although the host target cells differ and thus the effect [20] [22].

Other enzyme active subunits A from $\mathrm{AB}$ toxins elaborated by Corynebacterium diphtheria, Pseudomonas aeruginosa and Shigella dysenteriae can inactivate protein syntheses in the eukaryotic target cells. The Elongation factor- 2 is the intracellular target damaged by diphtheria toxin and the ribosome is the sensitive host tissue cell's target affected by Shiga toxin [10] [23].

Membrane receptors for many $\mathrm{AB}$ bacterial toxins are the same lipid-containing components, for example, gangliosides, that are used by hormone molecules [20] [22].

There are some pathogens that can produce different type of toxin molecules simultaneously. Many Gram-negative bacteria, for example, E. coli and V. cholera, are able to synthesis endotoxin LPS as well as exotoxin proteins such as enterotoxins and cytolysins. The set of toxins with different mechanism of damaging action is one of the reasons which cause unlike virulence potency in strain multiplicity.

The bacterial cell produces toxin molecules that are not toxic for the prokaryotic organism, but their damaging actions are aimed at eukaryotic cells. There is no direct connection between production of the toxin macromolecules and viability of pathogenic bacteria. Facts show that the capacity of exotoxin synthesis is caused by a biological stimulus to an increasing number of the microbial population.

\section{Genetic Control}

The study of the genetic bases of bacterial pathogenicity showed that appearance of the distinct genetic elements into the bacterial genome contributes to production of pathogenic determinants that are responsible for pathogenicity potency. These genomic regions, named "pathogenicity islands (PAI)", are acquired by means horizontal gene transfer mechanism [24]. They are found in pathogens that undergo gene transfer by plasmid, bacteriophage or conjugative transposon. For example, the major exo-protein toxins are secreted by bacteria under the action of the toxgenes containing into phages (cholera and cholera-like LT enterotoxins, diphtheria toxin) or plasmid (anthrax toxin). Sometimes PAI may be incorporated in the bacterial chromosome. It is known that the synthesis of LPS Gram-negative bacteria is encoded by genes located on the chromosome. It is quite possible that other pathogenicity factors are being as the somatic element of bacterial cell also encoded by the chromosome genes.

PAI are usually absent from non-pathogenic organisms of the same species. The gene combination in PAI causes the appearance of various bacterial strains that are distinguished in their virulence [25]. Apparently, the horizontal gene 
transfer mechanism may be qualified as an adaptive process that was used bacterial pathogen to conquest new ecological niche in multi-cellular organism.

\section{Discussion}

The use of molecular biology in the experimental study of infectious disease agent's pathogenicity brought us closer to understanding the biological nature of the phenomenon, based on the interaction of prokaryotic and eukaryotic organisms. Based on the definition of ecology as a science, an infectious disease can be considered a special model of an ecosystem, in which a living organism is a pathogen, and its natural environment is multi-cellular tissues of humans, animals or plants. In this habitat the microbe is able to perform its vital functions and interact with the tissue cells of its host's body. Death of the host's body as an outcome of infectious disease is a type of the ecological-like disaster, causing collapse of this agent's natural environment, followed by death of the majority of pathogen population [2].

Some biological functions that were acquired by bacteria during the occupation of new ecological niche in the multi-cellular host organism are fundament of the pathogenicity phenomenon.

The arsenal of pathogenicity factors allowed pathogens to colonize and replicate within hostile niches. It consists of bacterial adhesion to the cell surface and extracellular matrix of host tissue, cellular invasion by pathogens and bacterial evasion of host defenses.

We can assume that these pathogenicity functions were acquired by bacteria at the first stage of evolutionary process, and it lead to adapt germ in the new multi-cellular space. Their biological action was devoid of aggressive features and did not induce any damage to host multi-cellular systems. Close contacts between two types of cells, prokaryotic (bacteria) and eukaryotic (host), lead communicative events at the molecular level and development of damaging function as a result of this interaction. Apparently, under the action of the outside genetic information, new elements of non-chromosome heredity appeared in bacterial genome [26].

It is interesting to note the biological paradox in the bacterial pathogenicity phenomenon: the action of the first tree pathogenic functions (adhesion, invasion and evasion) is directed towards establishing an ecological niche in multicellular host, while the aim of the damaging factors is to disturb the space [27].

There are three approaches in consideration of pathogenicity phenomenon: biological, medical and applied aspects.

Biology of pathogenicity is based on the study of evolution, speciation of pathogens, ecology, genetic determination and encoding of biosynthesis of macromolecules possessing the function of pathogenicity factors (PF). The mechanism of interactions of PF and the host target-cells are also an important part in the biological process of pathogenicity.

The medical aspect of bacterial pathogenicity indicates a realization of pathogenic potency in the host organism. It is a problem of infectious disease, diag- 
nostic, prophylactic and treatment of illness induced by the pathogen.

In the applied area, the knowledge of PF, their structure and functions may have important practical implication such as providing delivery systems for vaccination, tools for cell biology, and tools for the development of new strategies for therapy of bacterial infections.

\section{Conclusion}

Systematic analysis of experimental data accumulated over the last two decades demonstrates the poly-functional feature of the phenomenon of bacterial pathogenicity. The main biological functions including adhesion, invasion, evasion, and damaging function are responsible for the interaction between the prokaryotic pathogen and the eukaryotic host tissue. The damaging function plays the leading role in the development of the disease specific syndrome. From an ecological point of view, the phenomenon of pathogenic bacteria can be considered as a factor in regulating the size of the population and at the same time the pathogen and the host organism. Since pathogenicity taxonomy position can be seen as a sign of the species, the virulence characteristic strains diverse within species.

\section{References}

[1] Ezepchuk, Yu.V. (1985) Pathogenicity as a Function of Biomolecules. Medicine, Moscow, 238 p.

[2] Ezepchuk, Yu.V. (2013) Biology of Pathogenicity (Theoretical Review). Annual Review \& Research in Biology, 3, 805-813.

[3] Beachey, E.H. (1981) Bacterial Adherence: Adhesin Receptor Interaction Mediating the Attachment of Bacteria to Mucosal Surface. The Journal of Infectious Diseases, 143, 325-345. https://doi.org/10.1093/infdis/143.3.325

[4] Kang, H.J., Baker, E.N. and Proft, T. (2012) Fimbrial Adhesins: Adhesive Molecules on a "Stalk". In: Locht, C. and Simonet, M., Eds., Bacterial Pathogenesis: Molecular and Cellular Mechanisms, Caister Academic Press, France, Chapter 4: 71-98.

[5] Sheets, A.J. and St. Geme III, J.W. (2012) Nonpilus (Non-Fimbrial) Adhesins. In: Locht, C. and Simonet, M., Eds., Bacterial Pathogenesis. Molecular and Cellular Mechanisms, Caister Academic Press, France, Chapter 5: 99-128.

[6] Wu, H.-J., Wang, A.H.-J. and Jennings, M.P. (2008) Discovery of Virulence Factors of Pathogenic Bacteria. Current Opinion in Chemical Biology, 12, 93-101. https://doi.org/10.1016/j.cbpa.2008.01.023

[7] Todar, K. (2009) The Mechanism of Bacterial Pathogenicity. The Microbial World, $1-26$.

[8] Popoff, M.R. and Bouvet, P. (2009) Clostridial Toxins. Future Microbiology, 4, 1024-1064. https://doi.org/10.2217/fmb.09.72

[9] Ezepchuk, Yu.V. (2012) Infectious Disease as Amolecular Model of Ecology-Like Disaster. Biotechnology, 5, 151-155.

[10] Wassenaar, T.M. (2012) Bacteria: The Benign, the Bad, and the Beautiful. WaleyBlackwell. Bacterial Toxins, 59-66; Enzymes, 67-74.

[11] Moreau, K. and Lafont, F. (2012) Mechanism of Bacterial Entry into Host Cells. In: Locht, C. and Simonet, M., Eds., Bacterial Pathogenesis. Molecular and Cellular 
Mechanisms, Caister Academic Press, France, Chapter, 9: 209-222.

[12] Furtado, A.R. and Subtil, A. (2012) The Bacterial Life in a Vacuole. In: Locht, C. and Simonet, M., Eds., Bacterial Pathogenesis: Molecular and Cellular Mechanisms, Caister Academic Press, France, Chapter 10: 223-242.

[13] Mostowy, S. and Cossart, P. (2012) The Bacterial Life in the Cytosol. In: Locht, C. and Simonet, M., Eds., Bacterial Pathogenesis. Molecular and Cellular Mechanisms, Caister Academic Press, France, Chapter 11: 243-260.

[14] Sara, M. and Steytr, U. (2000) S-Layer Proteins. Journal of Bacteriology, 182, 859868. https://doi.org/10.1128/JB.182.4.859-868.2000

[15] Hale, J.D.F. (2012) Bacterial Resistance to Antimicrobial Peptides. In: Locht, C. and Simonet, M., Eds., Bacterial Pathogenesis. Molecular and Cellular Mechanisms, Caister Academic Press, France, Chapter 14: 305-316.

[16] Kobayashi, S.D., Rigby, K.M. and DeLeo, F.R. (2012) Bacteria-Induced Host Cell Death. In: Locht, C. and Simonet, M., Eds., Bacterial Pathogenesis. Molecular and Cellular Mechanisms, Caister Academic Press, France, Chapter 15: 317-362.

[17] Salyer, A.A. and Whitt, D.D. (1994) Bacterial Pathogenesis. A Molecular Approach. ASM Press, Washington DC, 54-72.

[18] Sandvig, K., et al. (2010) Protein Toxins from Plants and Bacteria: Probes for Intracellular Transport and Tools in Medicine. FEBS Letters, 584, 2626-2634. https://doi.org/10.1016/j.febslet.2010.04.008

[19] Leung, D.Y.M., Huber, B.T. and Schlivert, P.M. (1997) Superantigens. Marcel Dekker, Inc., New York, 607.

[20] Jaljaszevicz, J. and Wadstrom, T. (1978) Bacterial Toxins and Cell Membranes. Academic Press, New York, 330.

[21] Alouf, J.E. (2012) Toxins Damaging Cellular Membranes: Paradigms and Molecular Features. In: Locht, C. and Simonet, M., Eds., Bacterial Pathogenesis. Molecular and Cellular Mechanisms, Caister Academic Press, France, Chapter 7: 171-180.

[22] Moayeri, M. and Leppla, S.H. (2009) Cellular and Systemic Effects of Anthrax Lethal Toxin and Edema Toxin. Molecular Aspects of Medicine, 30, 439-455. https://doi.org/10.1016/j.mam.2009.07.003

[23] Nang, W.J. and Guo, Q. (2009) The Adenylyl Cyclase Activity Anthrax Edema Factor. Molecular Aspects of Medicine, 30, 423-430. https://doi.org/10.1016/j.mam.2009.06.001

[24] Schmidt, H. and Hensel, M. (2006) Pathogenicity Islands in Bacterial Pathogenesis. Clinical Microbiology Reviews, 19, 257. https://doi.org/10.1128/CMR.19.1.257.2006

[25] Hacker, J. and Carniel, E. (2001) Ecology, Fitness, Genomic Islands and Bacterial Pathogenicity. A Darwinian View of the Evolution of Microbes. EMBO, 2, 376-381. https://doi.org/10.1093/embo-reports/kve097

[26] Ezepchuk, Yu.V. (2015) A Formula of Bacterial Pathogenicity (Theoretical Review). Annual Review \& Research in Biology, 5, 268-274.

[27] Bacterial Pathogenicity. http://www.pathogenicfactors.com 